TRANSACTIONS OF THE

AMERICAN MATHEMATICAL SOCIETY

Volume 351, Number 3, March 1999, Pages 1257-1275

S 0002-9947(99)02370-3

\title{
ON LOCALLY LINEARLY DEPENDENT OPERATORS AND DERIVATIONS
}

\author{
MATEJ BREŠAR AND PETER ŠEMRL
}

\begin{abstract}
The first section of the paper deals with linear operators $T_{i}$ : $U \longrightarrow V, i=1, \ldots, n$, where $U$ and $V$ are vector spaces over an infinite field, such that for every $u \in U$, the vectors $T_{1} u, \ldots, T_{n} u$ are linearly dependent modulo a fixed finite dimensional subspace of $V$. In the second section, outer derivations of dense algebras of linear operators are discussed. The results of the first two sections of the paper are applied in the last section, where commuting pairs of continuous derivations $d, g$ of a Banach algebra $\mathcal{A}$ such that $(d g)(x)$ is quasi-nilpotent for every $x \in \mathcal{A}$ are characterized.
\end{abstract}

\section{INTRODUCTION}

There are three different topics considered in this paper. The first two are almost entirely algebraic. Nevertheless, they might appear rather unrelated; the main reason why they are contained in the same paper is that they both are applied to the third topic, concerning derivations of Banach algebras.

We begin by describing the background concerning the first topic, which is treated in Section 2. Let $X$ be a complex vector space, $T$ be a linear operator from $X$ into $X$, and $n$ be a positive integer. Then $T$ is algebraic of degree less than or equal to $n$ if and only if for every $x \in X$ the vectors $x, T x, \ldots, T^{n} x$ are linearly dependent. Assume now that $X$ is a Banach space and $T$ a bounded operator. Then $T$ is algebraic if and only if for every $x \in X$ there exists a positive integer $n$ (depending on $x$ ) such that $x, T x, \ldots, T^{n} x$ are linearly dependent. These two statements on locally algebraic operators were proved by Kaplansky (see [8] and [12, p.63]). The first one has been generalized by Amitsur and Aupetit. Amitsur [1] considered linear operators $T_{1}, \ldots, T_{n}: U \rightarrow V$. Here, $U$ and $V$ are vector spaces over a field $F$. Let $V_{0}$ be a finite dimensional subspace of $V$. Amitsur proved that if $T_{1} u, \ldots, T_{n} u$ are linearly dependent modulo $V_{0}$ for every $u \in U$, then there exist $\alpha_{1}, \ldots, \alpha_{n} \in F$, not all zero, such that $S=\alpha_{1} T_{1}+\ldots+\alpha_{n} T_{n}$ satisfies

$$
\operatorname{dim} S U \leq \operatorname{dim} V_{0}+\left(\begin{array}{c}
n+1 \\
2
\end{array}\right)-1 .
$$

Aupetit $[2$, p. 86] considered the special case that $U$ and $V$ are complex vector spaces and $V_{0}=\{0\}$. He proved that in this special case $S$ can be chosen so that

$$
\operatorname{dim} S U \leq n-1 .
$$

Received by the editors February 12, 1997.

1991 Mathematics Subject Classification. Primary 15A04, 16W25, 47B47; Secondary 46H05, 47B 48 .

The authors were supported in part by the Ministry of Science of Slovenia.

(C)1999 American Mathematical Society 
Comparing these two results, we see that the assumptions in Amitsur's result are much more general. However, the estimate (2) is sharp, while this is not true for the inequality (1).

We will improve the result of Amitsur by obtaining a sharp estimate on rank of $S$ (Theorem 2.2). Our proof is considerably shorter and simpler than the proofs given by Amitsur and Aupetit. The only disadvantage of our approach is that we have to assume that $F$ is an infinite field. In fact, all our proofs work also when $F$ is a finite but sufficiently large field.

The converse of this result does not hold true. Namely, it is easy to find linear operators $T_{1}, \ldots, T_{n}: U \rightarrow V, u \in U$, and $\alpha_{1}, \ldots, \alpha_{n} \in F$ not all zero such that $\alpha_{1} T_{1}+\ldots+\alpha_{n} T_{n}$ has rank one while $T_{1} u, \ldots, T_{n} u$ are linearly independent. We will be able to obtain a complete description of locally linearly dependent operators in the case that $n=2$ (Theorem 2.3) and $n=3$ (Theorem 2.4). The problem of finding a similar characterization for larger values of $n$ seems to become extremely difficult. As we shall see later, it is impossible to solve this problem without knowing the description of all maximal subspaces of $n \times n$ matrices on which the determinant vanishes. The classification of all such subspaces is complicated even in the case that $n=4$ and $F$ is algebraically closed [6].

The assumption that $X$ is a Banach space is indispensable in the second mentioned result of Kaplansky (consider, for instance, the linear operator $f \mapsto f^{\prime}$ on a vector space of polynomials - it is not algebraic; however, for every polynomial $f$ there is $n$ such that $\left.f^{(n)}=0\right)$. Müller [9, Theorem 1] generalized the result of Kaplansky by proving an analogue of the Amitsur-Aupetit result for a countable family of locally linearly dependent bounded operators acting between two Banach spaces. We will conclude Section 2 by giving a short proof of this result.

Let us recall some basic definitions needed in Section 3. Let $X$ be a vector space and $\mathcal{L}(X)$ be the algebra of all linear operators on $X$. A set $\mathcal{S} \subset \mathcal{L}(X)$ is said to be dense on $X$ if for each finite linearly independent set $\left\{x_{1}, \ldots, x_{n}\right\} \subset X$ and arbitrary set $\left\{y_{1}, \ldots, y_{n}\right\} \subset X$ there exists a linear operator $S \in \mathcal{S}$ such that $S x_{i}=y_{i}, i=1, \ldots, n$. Recall that a derivation of an algebra $\mathcal{A}$ is a linear map $d$ of $\mathcal{A}$ into itself satisfying $d(S T)=d(S) T+S d(T)$ for all $S, T \in \mathcal{A}$. We say that a derivation $d$ of an algebra $\mathcal{A} \subset \mathcal{L}(X)$ is inner if $d(S)=[S, A]=S A-A S$ for some $A \in \mathcal{L}(X)$ (we do not require that $A$ lies in $\mathcal{A}$ ). Derivations that are not inner are called outer.

In Section 3 we treat outer derivations of subalgebras of $\mathcal{L}(X)$ that are dense on $X$. One might expect that it is much easier to use density when dealing with inner derivations than with the outer ones. Roughly speaking, we will show that this is not quite so. Namely, we will prove that given an outer derivation $d$ of a dense algebra $\mathcal{A}$, a linearly independent set $\left\{x_{1}, \ldots, x_{n}\right\} \subset X$ and arbitrary sets $\left\{y_{1}, \ldots, y_{n}\right\},\left\{z_{1}, \ldots, z_{n}\right\} \subset X$, then there exists $T \in \mathcal{A}$ such that $T x_{i}=y_{i}$, $d(T) x_{i}=z_{i}, i=1, \ldots, n$ (Theorem 3.6). The special case, when $n=2$, is already known [4]. We believe that this result could be a useful tool for reducing the problems concerning general derivations to the case of inner derivations. We illustrate how this reduction works in Section 4, where derivations of Banach algebras are treated. It is well-known that every continuous derivation of a Banach algebra $\mathcal{A}$ leaves primitive ideals of $\mathcal{A}$ invariant [13], and therefore it induces derivations on quotients of $\mathcal{A}$ by its primitive ideals. But these quotients can be represented as dense algebras of linear operators, so that the result of Section 3 can be used. 
The aim of Section 4 is to illustrate the applicability of the results of Sections 2 and 3 by characterizing commuting pairs $d, g$ of continuous derivations of a Banach algebra such that $d g(x)$ is quasi-nilpotent for every $x \in \mathcal{A}$. In particular, it turns out that $d g(x)^{3}$ must lie in the radical of $\mathcal{A}$ for every $x \in \mathcal{A}$. A special case of this problem, when $d=g$, was treated in [11] and [14] (more precisely, [11] deals only with inner derivations, while [14] also considers higher powers of derivations). On the other hand, we were motivated by a well-known ring-theoretic result of Posner [10] stating, in particular, that the product of two nonzero derivations of a prime ring of characteristic not 2 cannot be zero. Contrary to the result in the present paper, the commutativity of derivations is not required in Posner's theorem. We shall see, however, that the proof of the result of Section 4 is rather long and involved even under the assumption of commutativity.

\section{LOCALLY LINEARLY DEPENDENT OPERATORS}

We begin with a simple lemma which will play an important role in the sequel.

Lemma 2.1. Let $W$ be a vector space over a field $F, r$ a positive integer, $w_{1}, \ldots, w_{r}$ linearly independent vectors in $W$, and $z_{1}, \ldots, z_{r}$ arbitrary vectors in $W$. Then $w_{1}+\alpha z_{1}, \ldots, w_{r}+\alpha z_{r}$ are linearly independent for all but finitely many values of $\alpha \in F$.

Proof. Let $\mathcal{B}=\left\{w_{1}, \ldots, w_{r}, e_{1}, \ldots, e_{k}\right\}$ be a basis of the linear span of vectors $\left\{w_{1}, \ldots, w_{r}, z_{1}, \ldots, z_{r}\right\}$. For every $\alpha \in F$ we define an $r \times r$ matrix $A_{\alpha}$ whose $i$-th column consists of the first $r$ coordinates of the vector $w_{i}+\alpha z_{i}$ with respect to the basis $\mathcal{B}$. Obviously, $\operatorname{det} A_{0}=1$. Therefore, $\operatorname{det} A_{\alpha}=p(\alpha)$ is a nonzero polynomial in $\alpha$. If $p(\alpha) \neq 0$, then the vectors $w_{1}+\alpha z_{1}, \ldots, w_{r}+\alpha z_{r}$ are linearly independent. This completes the proof.

Theorem 2.2. Let $U$ and $V$ be vector spaces over an infinite field $F$ and let $V_{0}$ be a finite dimensional subspace of $V$. Let $T_{1}, \ldots, T_{n}: U \rightarrow V$ be linear operators. If $T_{1} u, \ldots, T_{n} u$ are linearly dependent modulo $V_{0}$ for every $u \in U$, then there exist $\alpha_{1}, \ldots, \alpha_{n} \in F$, not all zero, such that $S=\alpha_{1} T_{1}+\ldots+\alpha_{n} T_{n}$ satisfies

$$
\operatorname{dim} S U \leq \operatorname{dim} V_{0}+n-1 .
$$

This inequality is sharp.

Proof. If for all $u \in U$ the vectors $T_{1} u, \ldots, T_{n-1} u$ are linearly dependent modulo $V_{0}$, it is enough to prove the result with $T_{1}, \ldots, T_{n-1}$. So suppose that there exists $x \in U$ such that $T_{1} x, \ldots, T_{n-1} x$ are linearly independent modulo $V_{0}$. Then there exist $\alpha_{1}, \ldots, \alpha_{n-1} \in F$ such that $\left(T_{n}-\alpha_{1} T_{1}-\ldots-\alpha_{n-1} T_{n-1}\right) x \in V_{0}$. We define a linear operator $S$ by $S=T_{n}-\alpha_{1} T_{1}-\ldots-\alpha_{n-1} T_{n-1}$. Let $V_{1}$ be a subspace of $V$ generated by $V_{0}$ and $\left\{T_{1} x, \ldots, T_{n-1} x\right\}$. We choose a basis $\left\{f_{1}, \ldots, f_{q}\right\}$ of $V_{0}$. Then $T_{1} x, \ldots, T_{n-1} x, f_{1}, \ldots, f_{q}$ form a basis of $V_{1}$. In order to prove (3) we have to show that $S U \subset V_{1}$. Assume on the contrary that there exists $y \in U$ such that $T_{1} x, \ldots, T_{n-1} x, f_{1}, \ldots, f_{q}, S y$ are linearly independent. Since $F$ is infinite, it follows from Lemma 2.1 that we can find a nonzero $\alpha \in F$ such that

$$
T_{1} x+\alpha T_{1} y, \ldots, T_{n-1} x+\alpha T_{n-1} y, f_{1}, \ldots, f_{q}, S y=\alpha^{-1} S(x+\alpha y)-\alpha^{-1} S x
$$

are linearly independent. It follows that

$$
T_{1}(x+\alpha y), \ldots, T_{n-1}(x+\alpha y), S(x+\alpha y)
$$


are linearly independent modulo $V_{0}$ which further yields that

$$
T_{1}(x+\alpha y), \ldots, T_{n-1}(x+\alpha y), T_{n}(x+\alpha y)
$$

are linearly independent modulo $V_{0}$. This contradiction proves (3).

In order to see that the inequality (3) is sharp we consider a vector space $V=$ $V_{1} \oplus V_{0}$ with $\operatorname{dim} V_{1}=n-1$. Define $U$ as a direct sum of $n$ copies of $V$, and linear operators $T_{i}: U \rightarrow V$ by $T_{i}\left(v_{1} \oplus \ldots \oplus v_{n}\right)=v_{i}, i=1, \ldots, n$. Then $T_{1}, \ldots, T_{n}$ are locally linearly dependent modulo $V_{0}$, but every nontrivial linear combination of these operators has rank $n-1+\operatorname{dim} V_{0}$.

It is not difficult to give a complete description of a pair of locally linearly dependent operators. If $U$ and $V$ are linear spaces over $F, v$ a nonzero vector in $V$, and $f$ a nonzero linear functional on $U$, then $v \otimes f$ denotes the rank one operator mapping $U$ into $V$ defined by $(v \otimes f) z=f(z) v$ for all $z \in U$. Note that every operator of rank one can be written in this form.

Theorem 2.3. Let $U$ and $V$ be vector spaces over an infinite field $F$ and let $R, S$ : $U \rightarrow V$ be linear operators. Then the following are equivalent.

(a) The vectors $R u$ and $S u$ are linearly dependent for every $u \in U$.

(b) Either there exists $v \in V$ such that $R U \subset \operatorname{span}\{v\}$ and $S U \subset \operatorname{span}\{v\}$, or $R$ and $S$ are linearly dependent.

Proof. It is clear that (b) implies (a). So, assume that $R$ and $S$ are locally linearly dependent. First we will consider the case that one of them, say $R$, has rank one. Then $R$ can be written as $R=v \otimes f$ for some nonzero $v \in V$ and some nonzero linear functional $f$ on $U$. Let $w \in U$ be any vector satisfying $f(w)=1$. Assume that there exists $u \in U$ such that $S u$ and $v$ are linearly independent. We have $f(u)=0$, since otherwise $R u$ and $S u$ would be linearly independent. According to Lemma 2.1 there exists a nonzero $\alpha \in F$ such that $v=\alpha^{-1} R(u+\alpha w)$ and $S(u+\alpha w)$ are linearly independent. This contradiction shows that $S U \subset \operatorname{span}\{v\}$.

Let $R$ and $S$ be arbitrary locally linearly dependent operators. Then by Theorem 2.2 there exist $\alpha$ and $\beta$ in $F$ such that at least one of them, say $\beta$, is nonzero and $\alpha R+\beta S=T$ has rank at most one. If $T=0$ we are finished. So, let $T=v \otimes f$ be a rank one operator. Clearly, $R$ and $T$ are locally linearly dependent. Hence, $R U \subset \operatorname{span}\{v\}$, and consequently, $S U \subset \operatorname{span}\{v\}$. This completes the proof.

Before stating our result on three locally linearly dependent operators we will give an example of such operators. Let $v_{1}, v_{2}, v_{3}$ be linearly independent vectors in a vector space $V$, and let $Q_{1}$ and $Q_{2}$ be arbitrary $3 \times 3$ invertible matrices over a field $F, \operatorname{char} F \neq 2$. Assume further that $R$ is a linear mapping from $U$ into the space of all $3 \times 3$ skew-symmetric matrices. We define linear operators $R_{i}: U \rightarrow V$, $i=1,2,3$, by

$$
R_{i} u=\sum_{k=1}^{3}\left[Q_{1}(R u) Q_{2}\right]_{k i} v_{k} .
$$

Here, $\left[Q_{1}(R u) Q_{2}\right]_{k i}$ denotes the $(k, i)$-entry of the matrix $\left[Q_{1}(R u) Q_{2}\right]$. The computation

$$
\operatorname{det} R u=\operatorname{det}(R u)^{t r}=\operatorname{det}(-R u)=-\operatorname{det} R u
$$

shows that the matrix $Q_{1}(R u) Q_{2}$ is singular for every $u \in U$. This yields that $R_{1}, R_{2}, R_{3}$ are locally linearly dependent. 
Theorem 2.4. Let $U$ and $V$ be vector spaces over an infinite field $F$, $\operatorname{char} F \neq 2$, and let $R_{i}: U \rightarrow V, i=1,2,3$, be linear operators. Then the following are equivalent.

(i) The vectors $R_{1} u, R_{2} u$, and $R_{3} u$ are linearly dependent for every $u \in U$.

(ii) Either $R_{1}, R_{2}, R_{3}$ are linearly dependent, or there exist $v, w \in V$ such that $R_{i} U \subset \operatorname{span}\{v, w\}, i=1,2,3$, or there exist linearly independent vectors $v_{1}, v_{2}, v_{3} \in V, 3 \times 3$ invertible matrices $Q_{1}$ and $Q_{2}$, a linear mapping $R$ from $U$ into the space of all $3 \times 3$ skew-symmetric matrices such that $R_{i}$ has form (4), $i=1,2,3$, or there exists an idempotent $P: V \rightarrow V$ of rank one such that dim $\operatorname{span}\left\{\left(I_{V}-P\right) R_{1},\left(I_{V}-P\right) R_{2},\left(I_{V}-P\right) R_{3}\right\}=1$. Here, $I_{V}$ denotes the identity operator on $V$.

Proof. Once again, the implication (ii) $\Rightarrow(\mathrm{i})$ is trivial. So, assume that $R_{1}, R_{2}$, and $R_{3}$ are locally linearly dependent. According to Theorem 2.2 there exist scalars $\alpha_{i} \in F$, not all zero, such that $\alpha_{1} R_{1}+\alpha_{2} R_{2}+\alpha_{3} R_{3}=S$ has rank at most two. With no loss of generality we can assume that $\alpha_{3}=1$. If $S=0$ we are done. If $S \neq 0$ then $U$ and $V$ can be decomposed into direct sums $U=U_{1} \oplus U_{2}, V=V_{1} \oplus V_{2}$ with $\operatorname{rank} S=\operatorname{dim} U_{1}=\operatorname{dim} V_{1}$ such that $S$ has the matrix representation

$$
S=\left[\begin{array}{ll}
I & 0 \\
0 & 0
\end{array}\right]
$$

with respect to this direct sum decomposition. Here, $I$ stands for 1 in the case that $\operatorname{rank} S=1$ and for the $2 \times 2$ identity matrix in the case that $\operatorname{rank} S=2$. Let us denote the corresponding matrix representations of $R_{1}$ and $R_{2}$ by

$$
R_{1}=\left[\begin{array}{ll}
A_{1} & A_{2} \\
A_{3} & A_{4}
\end{array}\right] \text { and } R_{2}=\left[\begin{array}{cc}
B_{1} & B_{2} \\
B_{3} & B_{4}
\end{array}\right] .
$$

We will first prove that the operators

$$
\left[\begin{array}{cc}
0 & 0 \\
A_{3} & A_{4}
\end{array}\right] \text { and }\left[\begin{array}{cc}
0 & 0 \\
B_{3} & B_{4}
\end{array}\right]
$$

are locally linearly dependent. Assume on the contrary that there exists

$$
u=\left[\begin{array}{l}
u_{1} \\
u_{2}
\end{array}\right] \in U_{1} \oplus U_{2}
$$

such that $A_{3} u_{1}+A_{4} u_{2}$ and $B_{3} u_{1}+B_{4} u_{2}$ are linearly independent. Lemma 2.1 tells us that there is no loss of generality in assuming that $u_{1} \neq 0$. It is then easy to see that $R_{1} u, R_{2} u$, and $S u$ are linearly independent. This contradiction completes the first step of the proof.

According to the previous theorem we have two possibilities. The first one is that operators

$$
\left[\begin{array}{cc}
0 & 0 \\
A_{3} & A_{4}
\end{array}\right] \text { and }\left[\begin{array}{cc}
0 & 0 \\
B_{3} & B_{4}
\end{array}\right]
$$

map $U$ into a one-dimensional subspace of $V_{2}$. The second possibility is that these two operators are linearly dependent. In the first case we have $R_{i} U \subset V_{0} \subset V$, $i=1,2,3$, where $V_{0}$ is a subspace of dimension at most three. If the dimension of $V_{0}$ is at most two, we are done. So, assume that $\operatorname{dim} V_{0}=3$. We choose a basis in $V_{0}$. For every $u \in U$ we define a $3 \times 3$ matrix $R_{u}$ whose $i$-th column consists of the coordinates of $R_{i} u$ with respect to the chosen basis in $V_{0}$. Then $\left\{R_{u}: u \in U\right\}$ is a vector space of matrices with zero determinant. According to [6, p.265] there 
exist invertible $3 \times 3$ matrices $Q_{1}$ and $Q_{2}$ such that this vector space is contained in $Q_{1} \mathcal{M} Q_{2}$, where $\mathcal{M}$ is either the space of all skew-symmetric matrices, or the space of all matrices of the form

$$
\left[\begin{array}{lll}
0 & * & * \\
0 & * & * \\
0 & * & *
\end{array}\right],
$$

or the space of all matrices of the form

$$
\left[\begin{array}{lll}
* & * & * \\
0 & 0 & * \\
0 & 0 & *
\end{array}\right] .
$$

Operators $R_{1}, R_{2}, R_{3}$ have form (4) in the first case. In the second case they are linearly dependent while in the last case there exists an idempotent $P: V \rightarrow V$ of rank one such that $\operatorname{dim} \operatorname{span}\left\{\left(I_{V}-P\right) R_{1},\left(I_{V}-P\right) R_{2},\left(I_{V}-P\right) R_{3}\right\}=1$. Here, $I_{V}$ denotes the identity operator on $V$.

So, it remains to consider the case that

$$
\left[\begin{array}{cc}
0 & 0 \\
A_{3} & A_{4}
\end{array}\right] \text { and }\left[\begin{array}{cc}
0 & 0 \\
B_{3} & B_{4}
\end{array}\right]
$$

are linearly dependent. Then we can assume with no loss of generality that

$$
R_{2}=\left[\begin{array}{cc}
B_{1} & B_{2} \\
\delta A_{3} & \delta A_{4}
\end{array}\right]
$$

for some scalar $\delta$. If $\operatorname{rank} S=1$ we are finished. So, assume from now on that $\operatorname{rank} S=2$. Clearly, operators $R_{1}$,

$$
R_{2}^{\prime}=R_{2}-\delta R_{1}=\left[\begin{array}{cc}
C_{1} & C_{2} \\
0 & 0
\end{array}\right],
$$

and $S$ are locally linearly dependent. Applying Lemma 2.1 once again and using a similar approach as above, we see that either $A_{3}=0$ and $A_{4}=0$, or

$$
\left[\begin{array}{cc}
C_{1} & C_{2} \\
0 & 0
\end{array}\right] \text { and }\left[\begin{array}{cc}
I & 0 \\
0 & 0
\end{array}\right]
$$

are locally linearly dependent. In the first case we have $R_{i} U \subset V_{1}$ for $i=1,2,3$. In the second case Theorem 2.3 yields

$$
R_{2}^{\prime}=\left[\begin{array}{cc}
\sigma I & 0 \\
0 & 0
\end{array}\right]
$$

for some scalar $\sigma$. It follows easily that $R_{1}, R_{2}, R_{3}$ are linearly dependent. This completes the proof.

It follows from the above proof that we have to solve the classification problem for maximal vector spaces of $n \times n$ matrices with zero determinant if we want to get a complete description of $n$ locally linearly dependent operators. This classification problem has been solved only for $n \leq 4$ under the additional assumption that $F$ is algebraically closed [6, p.265].

The methods we have developed give a short and simple proof of the following generalization of Kaplansky's result due to Müller [9, Theorem 1]. 
Theorem 2.5. Let $X$ and $Y$ be real or complex Banach spaces, and let $T_{k}: X \rightarrow$ $Y, k=1,2, \ldots$, be bounded linear operators. Assume that for every $x \in X$ there exist an integer $n \geq 1$ (depending on $x$ ) such that $T_{1} x, \ldots, T_{n} x$ are linearly dependent. Then there exists an integer $k \geq 1$ and scalars $\alpha_{1}, \ldots, \alpha_{k}$, not all zero, such that $S=\alpha_{1} T_{1}+\ldots+\alpha_{k} T_{k}$ has finite rank.

Proof. Let $\mathcal{F}_{n}$ denote the set of all vectors $x \in X$ such that $T_{1} x, \ldots, T_{n} x$ are linearly dependent. Then $\bigcup_{n=1}^{\infty} \mathcal{F}_{n}=X$. Using a standard compactness argument (see for example [12, p.64]), it is possible to show that each $\mathcal{F}_{n}$ is closed. By the Baire category theorem there exists a positive integer $k$ such that $\mathcal{F}_{k}$ contains an open ball, say $\left\{x \in X:\left\|x-x_{0}\right\|<\varepsilon\right\}$. We will show that $\mathcal{F}_{k}=X$. Assume on the contrary that there exists $y \in Y$ such that $T_{1} y, \ldots, T_{k} y$ are linearly independent. It follows from Lemma 2.1 that the vectors $T_{1}\left(y+\alpha x_{0}\right), \ldots, T_{k}\left(y+\alpha x_{0}\right)$ are linearly independent for all but finitely many scalars $\alpha$. Hence, $T_{1}\left(x_{0}+(1 / \alpha) y\right), \ldots, T_{k}\left(x_{0}+\right.$ $(1 / \alpha) y)$ are linearly independent for all but finitely many nonzero scalars $\alpha$. This is impossible since $\mathcal{F}_{k}$ contains $\left\{x \in X:\left\|x-x_{0}\right\|<\varepsilon\right\}$. Hence, we have a desired relation $\mathcal{F}_{k}=X$. Using Theorem 2.2, we complete the proof.

\section{Outer DeRIVAtions of DENSE Algebras}

Throughout this section, $X$ will be a vector space over a field $F, \mathcal{A}$ will be a dense algebra of linear operators on $X$, and $d$ will be a derivation of $\mathcal{A}$. Our goal is to generalize the following result to the case when, instead of two, any finite number of vectors appear.

Lemma 3.1 ([4, Theorem 2.3]). Suppose that $d$ is outer. If $x_{1}, x_{2}$ are linearly independent vectors in $X$ and $y_{1}, y_{2}, z_{1}, z_{2}$ are arbitrary vectors in $X$, then there exists $T \in \mathcal{A}$ such that

$$
\begin{aligned}
T x_{1} & =y_{1}, & & T x_{2}=y_{2}, \\
d(T) x_{1} & =z_{1}, & & d(T) x_{2}=z_{2} .
\end{aligned}
$$

Lemma 3.2. Let $\mathcal{L}$ be a left ideal of $\mathcal{A}$ and $x, y$ be linearly independent vectors in $X$. Suppose that the vectors $T x$ and $T y$ are linearly dependent for every $T$ in $\mathcal{L}$. Then there exists $z \in \operatorname{span}\{x, y\}$ such that $z \neq 0$ and $\mathcal{L} z=0$.

Proof. Without loss of generality we may assume that $T_{0} y \neq 0$ for some $T_{0} \in \mathcal{L}$. By assumption we have $T_{0} x=\alpha T_{0} y$ for some $\alpha \in F$, that is, $T_{0}(x-\alpha y)=0$. Let us show that

$$
T(x-\alpha y)=0
$$

for every $T \in \mathcal{L}$. Pick $T \in \mathcal{L}$. Assume first that the vectors $T y, T_{0} y$ are linearly independent. In particular, $T y \neq 0$, so that $T x=\alpha_{T} T y$ for some $\alpha_{T} \in F$. Similarly, $\left(T+T_{0}\right) y=T y+T_{0} y \neq 0$, and so $\left(T+T_{0}\right) x=\alpha_{T+T_{0}}\left(T+T_{0}\right) y, \alpha_{T+T_{0}} \in F$. Hence

$$
\alpha_{T} T y+\alpha T_{0} y=T x+T_{0} x=\left(T+T_{0}\right) x=\alpha_{T+T_{0}} T y+\alpha_{T+T_{0}} T_{0} y .
$$

Since $T y$ and $T_{0} y$ are linearly independent, it follows that $\alpha_{T}=\alpha_{T+T_{0}}=\alpha$, proving (5).

Assume, therefore, that $T y$ and $T_{0} y$ are dependent. As $\mathcal{A} T_{0} \subset \mathcal{L}$ and $\mathcal{A} T_{0} y=X$, we can choose $T_{1} \in \mathcal{L}$ so that $T_{0} y$ and $T_{1} y$ are independent. Thus, $T_{1}(x-\alpha y)=0$. But then $\left(T+T_{1}\right) y$ and $T_{0} y$ are independent, so that $\left(T+T_{1}\right)(x-\alpha y)=0$. Consequently, $T(x-\alpha y)=0$. This proves the lemma. 
Let us remark that, at least when $F$ is infinite, Lemma 3.2 follows at once from Theorem 2.3 by considering locally linearly dependent mappings $T \mapsto T x$ and $T \mapsto T y$ of $\mathcal{L}$ into $X$.

The next lemma is an extension of a result of Sinclair [13, Theorem 3.3].

Lemma 3.3. Let $V$ be a finite dimensional subspace of $X$ and $x \in X, x \notin V$. If $d$ is outer then there exists $T \in \mathcal{A}$ such that $T|V=d(T)| V=0$ and $T x \neq 0$.

Proof. Sinclair's result that we just mentioned states that the lemma is true if the dimension of $V$ is 1 . Set $n=\operatorname{dim} V$. Proceeding by induction, we may assume that the lemma is true for all subspaces of dimension less than $n$.

Let $\left\{e_{1}, \ldots, e_{n}\right\}$ be a basis of $V$, and set $V_{0}=\operatorname{span}\left\{e_{1}, \ldots, e_{n-1}\right\}$ and $\mathcal{L}_{0}=\{T \in$ $\left.\mathcal{A}: T\left|V_{0}=d(T)\right| V_{0}=0\right\}$. Note that $\mathcal{L}_{0}$ is a left ideal of $\mathcal{A}$. We claim that there exists $T_{0} \in \mathcal{L}_{0}$ such that $T_{0} e_{n}$ and $T_{0} x$ are independent. Namely, if this were not true, then, by Lemma 3.2 , there would be a nonzero $y \in \operatorname{span}\left\{e_{n}, x\right\}$ such that $\mathcal{L}_{0} y=0$. Of course, $y \notin V_{0}$ and $T y=0$ for any $T \in \mathcal{L}_{0}$. However, this contradicts the induction hypothesis.

Set $y_{1}=T_{0} x, y_{2}=T_{0} e_{n}, y_{3}=d\left(T_{0}\right) e_{n}$. Thus, $y_{1}$ and $y_{2}$ are independent. Let us show that there is $S \in \mathcal{A}$ such that

$$
S y_{1} \neq 0, \quad S y_{2}=0, \quad d(S) y_{2}+S y_{3}=0 .
$$

Assume first that $y_{1}, y_{2}, y_{3}$ are dependent, that is, $y_{3}=\alpha_{1} y_{1}+\alpha_{2} y_{2}$ for some $\alpha_{1}, \alpha_{2} \in F$. Then, by Lemma 3.1, there exists $S \in \mathcal{A}$ such that $S y_{1} \neq 0, S y_{2}=$ 0 and $d(S) y_{2}=-\alpha_{1} S y_{1}$, meaning that $S$ satisfies (6). Assume, therefore, that $y_{1}, y_{2}, y_{3}$ are independent.

Let $\mathcal{L}_{2}$ be a left ideal $\left\{T \in \mathcal{A}: T y_{2}=d(T) y_{2}=0\right\}$. Suppose that $T y_{1}$ and $T y_{3}$ are dependent for any $T \in \mathcal{L}_{2}$. Then it follows from Lemma 3.2 that there exists a nonzero $z \in \operatorname{span}\left\{y_{1}, y_{3}\right\}$ satisfying $\mathcal{L}_{2} z=0$. Of course, $y_{2}$ and $z$ are independent, and $T y_{2}=d(T) y_{2}=0$ implies $T z=0$. But, again, this contradicts the induction hypothesis. Thus, there is $T_{1} \in \mathcal{A}$ such that $T_{1} y_{1}$ and $T_{1} y_{3}$ are independent, and $T_{1} y_{2}=d\left(T_{1}\right) y_{2}=0$. Now pick $T_{2} \in \mathcal{A}$ such that $T_{2} T_{1} y_{1} \neq 0$ and $T_{2} T_{1} y_{3}=0$. Then $S=T_{2} T_{1}$ satisfies $S y_{1} \neq 0, S y_{3}=0, S y_{2}=0$ and $d(S) y_{2}=0$. In particular, (6) holds.

Now let $S \in \mathcal{A}$ be any operator satisfying (6) and set $T=S T_{0}$. Then $T \in \mathcal{L}_{0}$, that is, $T\left|V_{0}=d(T)\right| V_{0}=0$. Next,

$$
\begin{gathered}
T e_{n}=S T_{0} e_{n}=S y_{2}=0 \\
d(T) e_{n}=d(S) T_{0} e_{n}+S d\left(T_{0}\right) e_{n}=d(S) y_{2}+S y_{3}=0 \\
T x=S T_{0} x=S y_{1} \neq 0 .
\end{gathered}
$$

Thus, $T|V=d(T)| V=0$ and $T x \neq 0$. This proves the lemma.

Lemma 3.4. Let $V$ be a finite dimensional subspace of $X$ and $x, y \in X, x \notin V$. If $d$ is outer then there exists $T \in \mathcal{A}$ such that $T|V=d(T)| V=0, T x=y$ and $d(T) x=0$.

Proof. Let $\mathcal{L}=\{T \in \mathcal{A}: T \mid V=d(T) V=0\}$. By Lemma 3.3 there is $T_{0} \in \mathcal{L}$ such that $T_{0} x \neq 0$. There is no loss of generality in assuming that $T_{0} x=y$ (otherwise pick $S \in \mathcal{A}$ such that $S T_{0} x=y$ and consider $\left.S T_{0}\right)$. Set $y^{\prime}=d\left(T_{0}\right) x$. We claim that there exists $S \in \mathcal{A}$ such that

$$
S y=y, d(S) y+S y^{\prime}=0 .
$$


In the case when $y$ and $y^{\prime}$ are independent, this follows at once from Lemma 3.1. If $y=0$, then we can choose $S=0$. In the case when $y^{\prime}=\alpha y$ for some $\alpha \in F$, we apply Lemma 3.1 to find $S \in \mathcal{A}$ satisfying $S y=y$ and $d(S) y=-\alpha y$. Thus, in any case there is $S$ such that (7) holds true.

Now, let $T=S T_{0}$. Then $T \in \mathcal{L}$ and

$$
\begin{gathered}
T x=S T_{0} x=y, \\
d(T) x=d(S) T_{0} x+S d\left(T_{0}\right) x=0 .
\end{gathered}
$$

The lemma is thereby proved.

Lemma 3.5. Let $V$ be a finite dimensional subspace of $X$ and $x, z \in X, x \notin V$. If $d$ is outer then there exists $T \in \mathcal{A}$ such that $T|V=d(T)| V=0, T x=0$ and $d(T) x=z$.

Proof. By Lemma 3.4 there exists $T_{0} \in \mathcal{A}$ such that $T_{0}\left|V=d\left(T_{0}\right)\right| V=0, T_{0} x=x$ and $d\left(T_{0}\right) x=0$. Next, Lemma 3.1 implies that there is $S \in \mathcal{A}$ satisfying $S x=0$ and $d(S) x=z$. Now, $T=S T_{0}$ satisfies $T|V=d(T)| V=0, T x=0$, and $d(T) x=$ $d(S) T_{0} x+S d\left(T_{0}\right) x=d(S) x=z$.

We are now in a position to prove the main result of this section.

Theorem 3.6. Let $\mathcal{A}$ be a dense algebra of linear operators on a vector space $X$, and let $d$ be a derivation of $\mathcal{A}$. Then the following are equivalent.

(i) $d$ is outer.

(ii) Given any linearly independent set $\left\{x_{1}, \ldots, x_{n}\right\} \subset X$ and arbitrary sets $\left\{y_{1}, \ldots, y_{n}\right\},\left\{z_{1}, \ldots, z_{n}\right\} \subset X$, there exists $T \in \mathcal{A}$ such that

$$
\begin{gathered}
T x_{1}=y_{1}, T x_{2}=y_{2}, \ldots, T x_{n}=y_{n}, \\
d(T) x_{1}=z_{1}, d(T) x_{2}=z_{2}, \ldots, d(T) x_{n}=z_{n} .
\end{gathered}
$$

Proof. Let us first show that (i) implies (ii). From Lemmas 3.4 and 3.5 it follows that for any $i \in\{1, \ldots, n\}$ there are $T_{i 1}, T_{i 2} \in \mathcal{A}$ satisfying

$$
\begin{array}{ll}
T_{i 1} x_{j}=\delta_{i j} y_{j}, \quad d\left(T_{i 1}\right) x_{j}=0, & j=1, \ldots, n, \\
T_{i 2} x_{j}=0, \quad d\left(T_{i 2}\right) x_{j}=\delta_{i j} z_{j}, & j=1, \ldots, n .
\end{array}
$$

But then $T=\sum_{i=1}^{n} T_{i 1}+T_{i 2}$ satisfies the desired relations.

The converse is simple. Suppose that $d$ is inner, i.e., $d=[., A]$ for some linear operator $A$ on $X$. Note that $T x=0$ and $T A x=0$ imply $d(T) x=0$, so that (ii) certainly does not hold.

Corollary 3.7. The range of an outer derivation of a dense algebra is dense.

\section{Derivations of Banach algebras}

The goal of this section is to prove

Theorem 4.1. Let $\mathcal{B}$ be a complex Banach algebra and $d, g$ be continuous derivations of $\mathcal{B}$. If $d$ and $g$ commute, then the following are equivalent.

(i) $d g(x)$ is quasi-nilpotent for any $x \in \mathcal{B}$.

(ii) $d g(x)^{3}$ lies in the radical of $\mathcal{B}$ for any $x \in \mathcal{B}$.

(iii) If $\pi$ is a continuous irreducible representation of $\mathcal{B}$ on a Banach space $X$, then there exist linear operators $A$ and $B$ on $X$ such that $\pi(d(x))=[\pi(x), A]$, $x \in \mathcal{B}, \pi(g(x))=[\pi(x), B], x \in \mathcal{B}, A B=B A=0$, and either $A^{2}=0$ or $B^{2}=0$, unless $\pi d=0$ or $\pi g=0$. 
Proof. First, assume that (iii) holds. Pick a continuous irreducible representation $\pi$ on a Banach space $X$ and let $A, B$ be corresponding linear operators on $X$. Given any $x \in \mathcal{B}$, we then have

$$
\pi(d(g(x)))=[\pi(g(x)), A]=[[\pi(x), B], A]=-(A \pi(x) B+B \pi(x) A)
$$

for $A B=B A=0$. Now, using that $A^{2}=0$ or $B^{2}=0$, it follows at once that $\pi\left(d g(x)^{3}\right)=\pi(d(g(x)))^{3}=0$. Of course, in the case when $\pi d=0$ or $\pi g=0$, this is trivially true. Thus, $d g(x)^{3}$ lies in the kernel of any continuous irreducible representation of $\mathcal{B}$, meaning that (ii) holds true.

Assuming (ii), we see that $d g(x)^{3}$ is quasi-nilpotent for any $x \in \mathcal{B}$. But then $d g(x)$ is quasi-nilpotent, too.

Thus, the only nontrivial implication is (i) $\Longrightarrow$ (iii). Assume, therefore, that (i) holds and that $\pi$ is a continuous irreducible representation of $\mathcal{B}$ on a Banach space $X$. Then $\mathcal{A}=\pi(\mathcal{B}) \subset \mathcal{B}(X)$ is a dense algebra of (bounded) linear operators on $X$. Define $D, G: \mathcal{A} \longrightarrow \mathcal{A}$ by $D(\pi(x))=\pi(d(x)), G(\pi(x))=\pi(g(x))$. These maps are well-defined, for the kernel of $\pi$ is invariant under any continuous derivation [13, Theorem 2.2]. Moreover, $D$ and $G$ are commuting derivations of $\mathcal{A}$ and $D G(\pi(x))=$ $\pi(d g(x))$ is quasi-nilpotent for any $x \in \mathcal{B}$. Assuming that $D \neq 0$ and $G \neq 0$, we now have to show that $D=[., A], G=[., B]$ with $A B=B A=0$ and either $A^{2}=0$ or $B^{2}=0$.

The proof is broken up into a series of lemmas. Using the results of Sections 2 and 3, we first reduce the problem to the case when $D$ and $G$ are inner. Let us mention that we will not use the commutativity of $D$ and $G$ in the proof of the lemma below.

Lemma 4.2. There exist linear operators $A$ and $B$ on $X$ such that $D(T)=[T, A]$, $G(T)=[T, B]$ for all $T \in \mathcal{A}$.

Proof. Let us assume that $D$ is outer.

Suppose there exist $T \in \mathcal{A}$ and $x \in X$ such that $T x=D(T) x=0$ and $G(T) x \neq$ 0 . No matter whether $G(T) x$ and $(D G)(T) x$ are independent or not, Theorem 3.6 shows that there is $S \in \mathcal{A}$ such that $D(S) G(T) x=x$ and $S(D G)(T) x=0$. But then

$$
(D G)(S T) x=(D G)(S) T x+D(S) G(T) x+G(S) D(T) x+S(D G(T)) x=x,
$$

meaning that 1 lies in the spectrum of a quasi-nilpotent $D G(S T)$. This contradiction shows that

$$
T \in \mathcal{A}, x \in X, T x=D(T) x=0 \Longrightarrow G(T) x=0 .
$$

Now suppose that there exist $S \in \mathcal{A}$ and $x \in X$ such that the vectors $y_{1}=S x, y_{2}=$ $D(S) x, y_{3}=G(S) x$ are independent. According to Theorem 3.6 there is $T \in \mathcal{A}$ such that

$$
T y_{1}=0, T y_{2}=0, T y_{3} \neq 0, D(T) y_{1}=0 .
$$

In view of (8) we then also have $G(T) y_{1}=0$. However,

$$
\begin{gathered}
T S x=T y_{1}=0 \\
D(T S) x=D(T) S x+T D(S) x=D(T) y_{1}+T y_{2}=0 \\
G(T S) x=G(T) S x+T G(S) x=G(T) y_{1}+T y_{3}=T y_{3} \neq 0,
\end{gathered}
$$


contradicting (8). Therefore, $S x, D(S) x, G(S) x$ are dependent for every $S \in \mathcal{A}$ and every $x \in X$. First fixing $S \in \mathcal{A}$, it follows from Theorem 2.2 that there are $\alpha(S), \beta(S), \gamma(S) \in \mathbb{C}$, not all zero, such that $\alpha(S) S+\beta(S) D(S)+\gamma(S) G(S)$ is an operator of rank at most 2 . If it is not zero, it follows that $\mathcal{A}$ contains nonzero finite rank operators, contradicting the outerness of $D$ (see, e.g., [7, p.87]). Therefore, $\alpha(S) S+\beta(S) D(S)+\gamma(S) G(S)=0$ for any $S \in \mathcal{A}$. That is, the linear operators $D, G$ and $I$ on $\mathcal{A}$ are locally linearly dependent. Applying Theorem 2.2 again, we see that $\alpha I+\beta D+\gamma G$ is a finite rank operator (more precisely, its rank is at most 2) for some $\alpha, \beta, \gamma \in \mathbb{C}$, not all zero. Set $H=\beta D+\gamma G$. Thus, $H$ is a derivation of $\mathcal{A}$ such that $F=\alpha I+H$ has finite rank, i.e., $\mathcal{V}=F(\mathcal{A})$ is a finite dimensional subspace of $\mathcal{A}$. We now argue similarly as in [3]. Suppose that $H \neq 0$. Then $H(U) H(V) \neq 0$ for some $U, V \in \mathcal{A}$. Namely, $H(\mathcal{A})^{2}=0$ yields $H(T) S H(T)=H(T S) H(T)-T H(S) H(T)=0$ for all $S, T \in \mathcal{A}$, and by density of $\mathcal{A}$ it follows easily that $H(T)=0$ for any $T \in \mathcal{A}$. Now, for any $S, T \in \mathcal{A}$ we have

$$
\begin{aligned}
S H(U) H(V) T & =(H(S U)-H(S) U)(H(V T)-V H(T)) \\
& \in(\mathcal{V}+\mathcal{V} U)(V \mathcal{V}+\mathcal{V}) .
\end{aligned}
$$

Since $\mathcal{V}+\mathcal{V} U$ and $V \mathcal{V}+\mathcal{V}$ are finite dimensional spaces, it is easy to see that $\mathcal{I}=\operatorname{span}\{S H(U) H(V) T: S, T \in \mathcal{A}\}$ is a a finite dimensional ideal of $\mathcal{A}$. As $\mathcal{I}$ itself is a dense algebra of operators on $X$ [7, p. 33], it follows easily that $X$ must be finite dimensional. But then $\mathcal{A}$ is isomorphic to the algebra of $k$ by $k$ matrices over $\mathbb{C}$, where $k$ is the dimension of $X$. However, as it is well-known, such an algebra does not admit outer derivations.

Therefore, $H=\beta D+\gamma G=0$. If $\beta=\gamma=0$, then $\alpha \neq 0$, so that $I$ is a finite rank operator, and again we have arrived at the matrix case. Since $D$ and $G$ are nonzero by assumption, we have $G=\lambda D$ for some nonzero $\lambda \in \mathbb{C}$. Consequently, $D^{2}(\mathcal{A})$ consists of quasi-nilpotent elements only. Pick a nonzero $x \in X$. In view of Theorem 3.6, there is $T \in \mathcal{A}$ such that $T x=0$ and $D(T) x=x$. Similarly, there is $S \in \mathcal{A}$ satisfying $D(S) x=x$ and $S D^{2}(T) x=0$. But then

$$
D^{2}(S T) x=D^{2}(S) T x+2 D(S) D(T) x+S D^{2}(T) x=2 x,
$$

showing that 2 lies in the spectrum of $D^{2}(S T)$ - a contradiction.

We have thereby shown that $D$ cannot be an outer derivation. A simple adaptation of the proof shows that $G$ cannot be outer.

Remark 4.3. Henceforth we assume that $D$ and $G$ commute. Let us point out that then $D$ and $G$, and consequently $A$ and $B$, appear symmetrically.

Remark 4.4. Note that $D(T)=[T, A-\lambda]$ for any scalar operator $\lambda$, and that a derivation $c D$, where $c$ is any nonzero complex number, satisfies the same assumptions as $D$. Therefore, whenever it will be suitable, we will replace $A$ by $A-\lambda$ or $c A$, and, of course, we will do the same with $B$.

Remark 4.5. Our basic assumption is that

$$
D G(T)=[[T, B], A]=T B A-A T B-B T A+A B T
$$

is quasi-nilpotent for every $T \in \mathcal{A}$. This yields that for any $x \in X, B A x \in$ $\operatorname{span}\{x, A x, B x\}$. Indeed, if this were not true for some $x \in X$, then there would exist $T \in \mathcal{A}$ such that $T A x=T B x=T x=0$ and $T B A x=x$, and so $D G(T) x=x$. In particular, this shows that $A, B, I$ and $B A$ are locally linearly dependent. 
Remark 4.6. Another simple observation is that $B y$ and $y$ are dependent for any $y \in \operatorname{Im} A \cap \operatorname{Ker} A$. Namely, otherwise there would exist $T \in \mathcal{A}$ such that $T y=0$ and $A T B y=y$, so that $[[T, B], A] y=-y$. Hence there is $\lambda \in \mathbb{C}$ such that $B y=\lambda y$ for any $y \in \operatorname{Im} A \cap \operatorname{Ker} A$ (see the next remark).

Remark 4.7. Suppose that linear operators $S$ and $T$ on a vector space $Y$ satisfy $S y \in \operatorname{span}\{T y\}$ for any $y \in Y$. In particular, $S$ and $T$ are locally linearly dependent. However, note that in this situation the only possibility is that $S=\lambda T$ for some scalar $\lambda$.

Lemma 4.8. $A$ and $B$ commute.

Proof. As $D G=G D$, we have $[[T, B], A]=[[T, A], B]$, that is, $[T,[A, B]]=0$ for every $T \in \mathcal{A}$. The density of $\mathcal{A}$ implies that $[A, B]=\delta$ is a scalar operator.

Assume first that $A, B, I$ are locally linearly dependent. Then their nontrivial linear combination is a finite rank operator by Theorem 2.2. This readily implies that $\delta=[A, B]$ has finite rank. Now, $\delta \neq 0$ implies that $X$ is finite dimensional and hence $\mathcal{A}$ is isomorphic to the matrix algebra, and that the identity is a commutator. But this is certainly impossible (consider, for instance, the trace).

Thus, we may assume that $A, B, I$ are locally linearly independent, while, by Remark 4.5, $A, B, I, B A$ are locally linearly dependent. As is clear from the proof of Theorem 2.2, this implies that $B A=\alpha A+\beta B+\gamma+C$, where $C$ has finite rank. Set $B^{\prime}=B-\alpha, A^{\prime}=A-\beta$. Then $B^{\prime} A^{\prime}=\sigma+C$ with $\sigma=\gamma+\alpha \beta$ and $A^{\prime} B^{\prime}=B^{\prime} A^{\prime}+\delta=\delta+\sigma+C$. Hence $(\delta+\sigma) A^{\prime}+C A^{\prime}=A^{\prime} B^{\prime} A^{\prime}=\sigma A^{\prime}+A^{\prime} C$, so that $\delta A^{\prime}$ has finite rank. But then $\delta=\left[A^{\prime}, B^{\prime}\right]$ has finite rank, which, as already observed above, gives $\delta=0$.

Lemma 4.9. Let $Y$ be a subspace of $X$ invariant under both $A$ and $B$. If $A^{2} \mid Y=0$, then there exists a scalar $\mu$ such that $A(B-\mu)|Y=(B-\mu) A| Y=0$.

Proof. Suppose there is $y \in Y$ such that $A B y \notin \operatorname{span}\{A y\}$. Then there is $T \in \mathcal{A}$ satisfying $T A y=0$ and $T A B y=-y$, yielding $[[T, B], A] A y=A y$. As $A y \neq 0$ (namely, otherwise $A B y=B A y$ should be 0 , too), such a $y$ cannot exist. Therefore, by Remark 4.7, there is $\mu \in \mathbb{C}$ such that $A B y=B A y=\mu A y$ for all $y \in Y$, proving the lemma.

Lemma 4.10. Let $Y$ be a subspace of $X$ invariant under both $A$ and $B$. If $A B \mid Y=$ 0 , then either $A^{2} \mid Y=0$ or $B^{2} \mid Y=0$.

Proof. Assume first that there are $x, y \in Y$ such that $A^{2} x$ and $B^{2} y$ are linearly independent. Then we can find $T \in \mathcal{A}$ such that $T A^{2} x=-y, T B^{2} y=-x$, $T A x \in Y$ and $T B y \in Y$. But then $[[T, B], A](A x+B y)=A x+B y$. Note that $A x+B y \neq 0$ for $A^{2} x=A(A x+B y)$. Therefore, such $x$ and $y$ cannot exist. That is, $A^{2} x$ and $B^{2} y$ are dependent for any $x, y \in Y$. If neither $A^{2}$ nor $B^{2}$ is zero on $Y$, then there is $w \in Y$ such that $A^{2} Y=B^{2} Y=\operatorname{span}\{w\}$. Note that there is $y \in Y$ such that $A^{2} y=\lambda w \neq 0$ and $B^{2} y=\mu w \neq 0$. However, picking $T \in \mathcal{A}$ such that $T w=y, T A y \in Y$ and $T B y \in Y$, we arrive at $[[T, B], A]^{2} A y=\lambda \mu A y$. With this contradiction the lemma is proved. 
Remark 4.11. Let $\hat{A}$ and $\hat{B}$ be $m$ by $m$ matrices, with $m \geq 3$, of the form

$$
\hat{A}=\left(\begin{array}{cccccc}
0 & 1 & 0 & 0 & \ldots & 0 \\
0 & 0 & 1 & 0 & \ldots & 0 \\
0 & 0 & 0 & 1 & \ldots & 0 \\
\vdots & \vdots & \vdots & \vdots & \ddots & \vdots \\
0 & 0 & 0 & 0 & \ldots & 1 \\
0 & 0 & 0 & 0 & \ldots & 0
\end{array}\right), \quad \hat{B}=\left(\begin{array}{ccccc}
\lambda_{1} & \lambda_{2} & \lambda_{3} & \ldots & \lambda_{m} \\
0 & \lambda_{1} & \lambda_{2} & \ldots & \lambda_{m-1} \\
0 & 0 & \lambda_{1} & \ldots & \lambda_{m-2} \\
\vdots & \vdots & \vdots & \ddots & \vdots \\
0 & 0 & 0 & \ldots & \lambda_{1}
\end{array}\right)
$$

Suppose that $[[\hat{T}, \hat{B}], \hat{A}]$ is nilpotent for every $m$ by $m$ matrix $\hat{T}$. We claim that then $\lambda_{2}=\lambda_{3}=\ldots=\lambda_{m-1}=0$. Let us prove this by induction on $m$. When $m=3$, one can certainly prove this directly; on the other hand, the proof is implicitly given in the first part of the proof of the next lemma. Assume now that $m>3$ and that our claim is true for $m-1$. Picking any matrix $\hat{T}$ whose $m$-th row and $m$-th column are 0 , and using the induction assumption, we see easily that $\lambda_{2}=\ldots=\lambda_{m-2}=0$. Letting $\hat{T}=E_{m 1}$ (a matrix unit), we get $\lambda_{m-1}=0$.

Lemma 4.12. Let $Y$ be a subspace of $X$ invariant under both $A$ and $B$. If there exists an integer $n \geq 2$ such that $A^{n} \mid Y=0$ and $A^{n-1} \mid Y \neq 0$, then there is a scalar $\mu$ such that $A(B-\mu) \mid Y=0$. Moreover, if $n>2$, then $(B-\mu)^{2} \mid Y=0$.

Proof. Lemma 4.9 states that the result is true when $n=2$. Let us now consider the case when $n=3$.

We have $A^{2}(Y) \subset \operatorname{Im} A \cap \operatorname{Ker} A$. Therefore, there is $\lambda \in \mathbb{C}$ such that $B A^{2} y=\lambda A^{2} y$ for every $y \in Y$ (Remark 4.6). Replacing $B$ by $B-\lambda$ we see that there is no loss of generality in assuming that $\lambda=0$, that is, $B A^{2} \mid Y=0$.

Set $Y_{1}=B(Y)$ and observe that $Y_{1}$ is invariant under both $A$ and $B$, and that $A^{2} \mid Y_{1}=0$. Lemma 4.9 tells us that there is $\mu \in \mathbb{C}$ such that $(B-\mu) A \mid Y_{1}=0$, that is, $\left(B^{2} A-\mu B A\right) \mid Y=0$.

Assume first that $\mu=0$. We claim that then $A B \mid Y=0$. Indeed, if $A B y \neq 0$ for some $y \in Y$, then $[[T, B], A] A B y=A B y$ for any $T \in \mathcal{A}$ satisfying $T A B y=y$. The final conclusion now follows from Lemma 4.10.

Suppose, therefore, that $\mu \neq 0$. There is no loss of generality in assuming that $\mu=1$, i.e., $B^{2} A|Y=B A| Y$. Suppose that $x \in Y$ is such that $A^{2} x \neq 0$ and $A B x \neq 0$. Recall that $A B x=\alpha A x+\beta B x+\gamma x$ for some $\alpha, \beta, \gamma \in \mathbb{C}$ (Remark 4.5). Since $B A^{2} x=0$ and $A^{3} x=0$, it follows that $\gamma A^{2} x=0$, and so $\gamma=0$. Next we have $0=B^{2} A^{2} x=\alpha B A^{2} x+\beta B^{2} A x=\beta B A x$, so that $\beta=0$. Finally, we have $0=B A^{2} x=\alpha A^{2} x$. Hence $\alpha=0$, which further implies $A B x=0$, contrary to the assumption. This proves that for every $x \in Y$ we have either $A^{2} x=0$ or $A B x=0$. Since $A^{2} \mid Y \neq 0$, it follows that $A B \mid Y=0$. Applying Lemma 4.10, we now see that the lemma is proved for $n=3$.

Now let $n>3$. Choose $x \in Y$ with $A^{n-1} x \neq 0$. By Remark 4.6 we have $B A^{n-1} x \in \operatorname{span}\left\{A^{n-1} x\right\}$. We claim that $B A^{k} x$ lies in

$$
\operatorname{span}\left\{A^{k} x, A^{k+1} x, \ldots, A^{n-1} x\right\},
$$

$k=n-1, n-2, \ldots, 1$. As this is true for $k=n-1$, we may assume that it is true for $n-1, n-2, \ldots, k$ with $k>1$, and let us prove that then it is also true for $k-1$. Indeed, if this were not true, then there would be $T \in \mathcal{A}$ satisfying $T B A^{k-1} x=$ $A^{k-2} x$ and $T B A^{k} x=T A^{k} x=T A^{k-1} x=0$, so that $[[T, B], A] A^{k-1} x=-A^{k-1} x-$ a contradiction. Hence it follows that $W=\operatorname{span}\left\{A x, A^{2} x, \ldots, A^{n-1} x\right\}$ is invariant under both $A$ and $B$. Note that the restrictions of $A$ and $B$ to $W$ with respect 
to the basis $\left\{A^{n-1} x, A^{n-2} x, \ldots, A x\right\}$ have matrix representations $\hat{A}$ and $\hat{B}$ (where $m=n-1)$, respectively. Note that the conclusion of Remark 4.11 can be applied, i.e., $\lambda_{2}=\lambda_{3}=\ldots=\lambda_{n-2}=0$. It follows that $B A^{2} x=\lambda_{1} A^{2} x$.

We claim that $B A^{2} \mid Y$ and $A^{2} \mid Y$ are locally linearly dependent. Suppose this is not true, i.e., $B A^{2} y$ and $A^{2} y$ are independent for some $y \in Y$. By Lemma 2.1 it follows that $B A^{2}(y+\alpha x)$ and $A^{2}(y+\alpha x)$ are independent for all but finitely many $\alpha \in \mathbb{C}$. But, by what we have just proved, this implies that $A^{n-1}(y+\alpha x)=0$ for all but finitely many $\alpha \in \mathbb{C}$. However, then $A^{n-1} x=0$, contrary to the assumption.

Now, Theorem 2.3 tells us that we have two possibilities. The first one is that $B A^{2}(Y)=A^{2}(Y)=\operatorname{span}\{w\}$ for some $w \in Y$. In particular, $A w \in \operatorname{span}\{w\}$. But since $A \mid Y$ is nilpotent, we must have $A w=0$ and so $A^{3} \mid Y=0$. This, of course, contradicts the assumption that $n>3$. The second possibility is that $B A^{2}\left|Y=\lambda A^{2}\right| Y$ for some $\lambda \in \mathbb{C}$. Again, without loss of generality we may assume that $\lambda=0$. Let $Y_{1}=A(Y)$. Then $A B \mid Y_{1}=0$. Since $A^{3} \mid Y \neq 0$, it follows from Lemma 4.10 that $B^{2} \mid Y_{1}=0$, that is, $B^{2} A \mid Y=0$. This implies that if $T \in \mathcal{A}$ satisfies $T A B y=y$ for some $y \in Y$, then $[[T, B], A] A B y=A B y$. Therefore, $A B \mid Y=0$. Now apply Lemma 4.10, and the proof is complete.

Lemma 4.13. Let $Y$ be a finite dimensional subspace of $X$ invariant under both $A$ and $B$. Then there exist scalars $\lambda$ and $\mu$ such that $(A-\lambda)(B-\mu) \mid Y=0$ and either $(A-\lambda)^{2} \mid Y=0$ or $(B-\mu)^{2} \mid Y=0$.

Proof. First of all, note that we may assume that $A$ and $B$ are matrices. More precisely, it suffices to prove the conclusion of the lemma under the assumption that $A, B$ are commuting $n$ by $n$ complex matrices such that $[[T, B], A]$ is a nilpotent matrix for every $T \in M_{n}$, the algebra of $n$ by $n$ complex matrices. In view of Lemma 4.12 it is enough to show that either $A$ or $B$ has only one eigenvalue.

There is nothing to prove when $n=1$. Let $n=2$ and suppose that both $A$ and $B$ have two distinct eigenvalues. Then they are diagonalizable, and since they commute there is no loss of generality (Remark 4.4) in assuming that $A=\lambda E_{11}$ and $B=\mu E_{22}$ for some (nonzero) $\lambda, \mu \in \mathbb{C}$. However, then $A B=0$, so that Lemma 4.10 can be applied.

We proceed by induction on $n$. Assume that the lemma is true for every positive integer smaller than $n$, but is not true for $n$. Without loss of generality we then have

$$
A=\left(\begin{array}{cc}
A_{1} & 0 \\
0 & A_{2}
\end{array}\right)
$$

with $A_{1}$ and $A_{2}$ not having a common eigenvalue. Since $A$ and $B$ commute, using Rosenblum's corollary [12, Corollary 0.13$]$ we see that

$$
B=\left(\begin{array}{cc}
B_{1} & 0 \\
0 & B_{2}
\end{array}\right)
$$

If $B_{1}$ has more than one eigenvalue, we have

$$
B_{1}=\left(\begin{array}{cc}
B_{3} & 0 \\
0 & B_{4}
\end{array}\right)
$$

with $B_{3}$ and $B_{4}$ not having a common eigenvalue. Hence

$$
A=\left(\begin{array}{ccc}
A_{3} & 0 & 0 \\
0 & A_{4} & 0 \\
0 & 0 & A_{2}
\end{array}\right), \quad B=\left(\begin{array}{ccc}
B_{3} & 0 & 0 \\
0 & B_{4} & 0 \\
0 & 0 & B_{2}
\end{array}\right)
$$


where at least one of the matrices

$$
\left(\begin{array}{cc}
B_{3} & 0 \\
0 & B_{2}
\end{array}\right), \quad\left(\begin{array}{cc}
B_{4} & 0 \\
0 & B_{2}
\end{array}\right)
$$

has at least two distinct eigenvalues. With no loss of generality we may assume that this is true for the first matrix. Now set

$$
T=\left(\begin{array}{ccc}
T_{1} & 0 & T_{2} \\
0 & 0 & 0 \\
T_{3} & 0 & T_{4}
\end{array}\right)
$$

and note that the fact that $[[T, B], A]$ is nilpotent implies that

$$
\left[\left[\left(\begin{array}{cc}
T_{1} & T_{2} \\
T_{3} & T_{4}
\end{array}\right),\left(\begin{array}{cc}
B_{3} & 0 \\
0 & B_{2}
\end{array}\right)\right],\left(\begin{array}{cc}
A_{3} & 0 \\
0 & A_{2}
\end{array}\right)\right]
$$

is nilpotent. By the induction hypothesis we see that at least one of the matrices

$$
\left(\begin{array}{cc}
B_{3} & 0 \\
0 & B_{2}
\end{array}\right), \quad\left(\begin{array}{cc}
A_{3} & 0 \\
0 & A_{2}
\end{array}\right)
$$

has exactly one eigenvalue - a contradiction. Therefore, $B_{1}$, and similarly $B_{2}$, cannot have two distinct eigenvalues. That is, each of them has exactly one eigenvalue, but these two eigenvalues are distinct. As $n \geq 3$, there is no loss of generality in assuming that $A_{1}$ and $B_{1}$ are commuting $k$ by $k$ matrices with $k \geq 2$, and that there is a nontrivial proper subspace $Y_{1}$ invariant under both $A_{1}$ and $B_{1}$. Now apply the induction assumption for the operators $A_{1} \mid Y_{1} \oplus A_{2}$ and $B_{1} \mid Y_{1} \oplus B_{2}$, which both have at least two eigenvalues. This proves the lemma.

Lemma 4.14. If both $A$ and $B$ are algebraic, then there exist scalars $\lambda$ and $\mu$ such that $(A-\lambda)(B-\mu)=0$ and either $(A-\lambda)^{2}=0$ or $(B-\mu)^{2}=0$.

Proof. Let $x \in X$. Then $\operatorname{span}\left\{A^{m} B^{n} x: m, n \geq 0\right\}$ is a finite dimensional subspace of $X$ invariant under both $A$ and $B$. Therefore, Lemma 4.13 implies that for any $x \in X$ either $x, A x, A^{2} x$ are dependent or $x, B x, B^{2} x$ are dependent. Suppose there exist $y, z \in X$ such that $y, A y, A^{2} y$ are independent and $z, B z, B^{2} z$ are independent. However, Lemma 2.1 then implies that there is $\alpha \in \mathbb{C}$ such that $y+\alpha z$, $A(y+\alpha z), A^{2}(y+\alpha z)$ as well as $y+\alpha z, B(y+\alpha z), B^{2}(y+\alpha z)$ are independent we have just shown that this is impossible. This proves that either $x, A x, A^{2} x$ are dependent for any $x \in X$, or that this is true for $B$. Of course, we may assume that this holds true for $A$. By Kaplansky's theorem on locally algebraic operators it follows that there is a polynomial $p$ of degree at most 2 such that $p(A)=0$. Of course, we may assume that it is of degree 2 , for otherwise $A$ would be a scalar. Next, in view of Lemma 4.9 we may assume that $p$ has two distinct roots. But then there is no loss of generality in assuming that

$$
A=\left(\begin{array}{ll}
I & 0 \\
0 & 0
\end{array}\right)
$$

and (since $A$ and $B$ commute) that

$$
B=\left(\begin{array}{cc}
B_{1} & 0 \\
0 & B_{2}
\end{array}\right) \text {. }
$$

Let $X=X_{1} \oplus X_{2}$ be a corresponding decomposition of $X$. If $B_{1}=\lambda$ is a scalar, then $A(B-\lambda)=0$, so that Lemma 4.10 gives the desired conclusion. The same is true when $B_{2}=\mu$ is a scalar, for then $(A-I)(B-\mu)=0$. Thus, it remains to treat 
the case when there exist $u \in X_{1}$ and $v \in X_{2}$ such that $B_{1} u$ and $u$ are independent, and $B_{2} v$ and $v$ are independent. But then there is $T \in \mathcal{A}$ such that $T u=T v=0$, $T B_{1} u=-v$ and $T B_{2} v=u$, which yields $[[T, B], A](u+v)=-(u+v)$. With this contradiction the lemma is proved.

Lemma 4.15. If $A$ has a finite rank, then there exist scalars $\lambda$ and $\mu$ such that $(A-\lambda)(B-\mu)=0$ and either $(A-\lambda)^{2}=0$ or $(B-\mu)^{2}=0$.

Proof. In view of Lemma 4.13 we may assume that $X$ is infinite dimensional. Therefore, 0 lies in the spectrum of $A$. Also, according to Lemma 4.12 there is no loss of generality in assuming that $A$ is not nilpotent, so that the spectrum of $A$ contains at least one nonzero point.

We shall prove the lemma by induction on $n=\operatorname{rank} A$. Suppose $n=1$. As $A$ is not nilpotent, it is a scalar multiple of the projection; with no loss of generality we may assume that $A$ is a rank one projection. As $A$ and $B$ commute, it follows at once that $A B=\mu A$ for some $\mu \in \mathbb{C}$. Replacing $B$ by $B-\mu$ and using Lemma 4.10 , we get the desired conclusion for $n=1$. Now suppose that the lemma is true whenever the rank of $A$ is smaller than $n$. As $A$ has finite rank, it has a matrix representation

$$
A=\left(\begin{array}{cc}
A_{1} & 0 \\
0 & N
\end{array}\right)
$$

where $A_{1}$ is an invertible operator (on a finite dimensional space), and $N$ is nilpotent. Since $A$ and $B$ commute, we have

$$
B=\left(\begin{array}{cc}
B_{1} & 0 \\
0 & B_{2}
\end{array}\right)
$$

Let $X=X_{1} \oplus X_{2}$ be a decomposition corresponding to these matrix representations. As $A$ is not nilpotent, $\operatorname{dim} X_{1} \geq 1$.

Suppose first that $\operatorname{dim} X_{1}=1$. Without loss of generality we may assume that

$$
A=\left(\begin{array}{cc}
1 & 0 \\
0 & N
\end{array}\right), \quad B=\left(\begin{array}{cc}
b_{1} & 0 \\
0 & B_{2}
\end{array}\right) .
$$

Also, by Lemma 4.12 we may assume that $N B_{2}=0$. If $b_{1}=0$, then Lemma 4.10 finishes the proof. With no loss of generality we may therefore assume that $b_{1}=1$.

We claim that for any $z \in X_{2}$ we have $B_{2}\left(I-B_{2}\right) z \in \operatorname{span}\left\{\left(I-B_{2}\right) z, N z\right\}$. Suppose this is not true for some $z \in X_{2}$. Set $v=z-B_{2} z$. Then $v \neq 0$ and $N z=N\left(I-B_{2}\right) z=N v$. Hence $B_{2} v \notin \operatorname{span}\{v, N v\}$. Now pick $T \in \mathcal{A}$ such that

$$
\begin{gathered}
T\left(\begin{array}{c}
1 \\
0
\end{array}\right)=\left(\begin{array}{c}
\frac{1}{4} \\
z
\end{array}\right), \quad T\left(\begin{array}{c}
0 \\
v
\end{array}\right)=\left(\begin{array}{c}
0 \\
0
\end{array}\right), \\
T\left(\begin{array}{c}
0 \\
N v
\end{array}\right)=\left(\begin{array}{c}
0 \\
0
\end{array}\right), \quad T\left(\begin{array}{c}
0 \\
B_{2} v
\end{array}\right)=-\left(\begin{array}{c}
1 \\
z
\end{array}\right),
\end{gathered}
$$

and note that

$$
(A B T-A T B-B T A+T B A)\left(\begin{array}{l}
1 \\
v
\end{array}\right)=\left(\begin{array}{l}
1 \\
v
\end{array}\right),
$$

a contradiction. This shows that for any $z \in X_{2}$ the vectors $z, B_{2} z, B_{2}^{2} z$ are linearly dependent modulo $\operatorname{Im} N$. But then, according to Theorem 2.2, $B_{2}$ is algebraic, which in turn implies that $B$ is algebraic. Now apply Lemma 4.14. 
Suppose, therefore, that the dimension of $X_{1}$ is greater than 1 . Since $A_{1}$ and $B_{1}$ commute, they have a common invariant subspace $Y$ such that $Y \neq\{0\}$ and $Y \neq X_{1}$. We can now apply the induction hypothesis for the restrictions of $A$ and $B$ to $Y \oplus X_{2}$. As the spectrum of $A \mid Y \oplus X_{2}$ contains at least two points, we have $\left((A-\lambda) \mid Y \oplus X_{2}\right)^{2} \neq 0$ for any $\lambda \in \mathbb{C}$. Consequently, $\left((B-\mu) \mid Y \oplus X_{2}\right)^{2}=0$ for some $\mu \in \mathbb{C}$. This yields $\left(B_{2}-\mu\right)^{2}=0$, and so, again, $B$ is algebraic and Lemma 4.14 can be applied.

We now complete the proof of Theorem 4.1 by showing that the conclusion of the last two lemmas holds without any restrictions on $A$ and $B$.

Lemma 4.16. There exist scalars $\lambda$ and $\mu$ such that $(A-\lambda)(B-\mu)=0$ and either $(A-\lambda)^{2}=0$ or $(B-\mu)^{2}=0$.

Proof. Let us first consider the case when $I, A$ and $B$ are locally linearly dependent. By Lemma 4.13 we may assume that $X$ is infinite dimensional (that is, $I$ has infinite rank). Therefore, Theorem 2.4 shows that we have two possibilities. The first one is that there is an idempotent $P$ of rank 1 such that $\operatorname{span}\{I-P,(I-P) A,(I-P) B\}$ is one dimensional. Thus, $A-P A=\alpha(I-P)$ for some $\alpha \in \mathbb{C}$, and so $A=\alpha I+F$ with $F$ having a finite rank. But then $A$ is algebraic. Similarly, $B$ is algebraic, and we get the result from Lemma 4.14. The second possibility is that $I, A, B$ are dependent. It is easy to see that in this case we can assume without losing generality that $[[T, A], A]$ is quasi-nilpotent for any $T \in \mathcal{A}$. This case, however, is fairly easy and has already been treated in $[11,14]$. Nevertheless, a brief independent argument can be given by saying that $A$ must be algebraic by Remark 4.5 and Kaplansky's theorem on locally algebraic operators, so that Lemma 4.14 yields the desired conclusion.

Therefore, we may assume that there is $x \in X$ such that $x, A x, B x$ are linearly independent. Using Remark 4.5 and Theorem 2.2, together with its proof, we see that there exist $\alpha, \beta, \gamma \in \mathbb{C}$ such that $\operatorname{Im}(A B-\alpha A-\beta B+\gamma I) \subset \operatorname{span}\{x, A x, B x\}$. Let $W$ be a closed subspace of $X$ such that $X=\operatorname{span}\{x, A x, B x\} \oplus W$. Let $P \in \mathcal{B}(X)$ be a projection such that $\operatorname{Im} P=W$ and $\operatorname{Ker} P=\operatorname{span}\{x, A x, B x\}$.

Let us first consider the case when $P, P A, P B$ are not locally linearly dependent. We claim that in this case $A B-\alpha A-\beta B+\gamma I=0$. Suppose this is not true, that is, $(A B-\alpha A-\beta B+\gamma I) z \neq 0$ for some $z \in X$. By assumption, there is $y \in X$ such that $P y, P A y$ and $P B y$ are independent. By Lemma 2.1 there is a nonzero $\lambda \in \mathbb{C}$ such that $(A B-\alpha A-\beta B+\gamma I)(y+\lambda z) \neq 0$ and $P(y+\lambda z), P A(y+\lambda z), P B(y+\lambda z)$ are independent. Since $I, A, B$ and $A B$ are locally linearly dependent, so are $I, A, B$ and $A B-\alpha A-\beta B+\gamma I$. Thus, there exist $\tau_{1}, \tau_{2}, \tau_{3}, \tau_{4} \in \mathbb{C}$, not all zero, such that

$$
\begin{aligned}
& \tau_{1}(y+\lambda z)+\tau_{2} A(y+\lambda z)+\tau_{3} B(y+\lambda z) \\
& \quad+\tau_{4}(A B-\alpha A-\beta B+\gamma I)(y+\lambda z)=0 .
\end{aligned}
$$

Multiplying this relation from the left by $P$ and using $P(A B-\alpha A-\beta B+\gamma I)=0$, we see that $\tau_{1}=\tau_{2}=\tau_{3}=0$. But then $\tau_{4} \neq 0$ and so $(A B-\alpha A-\beta B+\gamma I)(y+\lambda z)=0$, contradicting the choice of $\lambda$. Thus, we indeed have $A B-\alpha A-\beta B+\gamma I=0$. Replacing $A$ by $A-\beta$ and $B$ by $B-\alpha$, we see that $A B$ is a scalar, say $A B=\delta$. As $x, A x, B x$ are independent, there is $T \in \mathcal{A}$ such that $T x=x, T A x=T B x=0$ and so $[[T, B], A] x=2 \delta x$. Therefore, $\delta=0$. Now complete the proof by Lemma 4.10.

Therefore, we may assume that $P, P A, P B$ are locally linearly dependent. Again, Theorem 2.4 shows that we have two possibilities. The first one is that there is a 
projection $Q$ of rank 1 such that $\operatorname{span}\{(I-Q) P,(I-Q) P A,(I-Q) P B\}$ is one dimensional. Therefore, $P A-Q P A=\sigma(P-Q P)$ for some $\sigma \in \mathbb{C}$, and so

$$
\begin{aligned}
A & =P A+(I-P) A=Q P A+\sigma P-\sigma Q P+(I-P) A \\
& =\sigma I+Q(P A-\sigma P)+(I-P)(A-\sigma I) .
\end{aligned}
$$

Since $Q(P A-\sigma P)+(I-P)(A-\sigma I)$ has finite rank, it follows that $A$ is algebraic. Similarly, $B$ is algebraic, and the result follows from Lemma 4.14.

The other possibility is that $P, P A$ and $P B$ are linearly dependent. That is, $\delta_{1} P+\delta_{2} P A+\delta_{3} P B=0$ for some $\delta_{1}, \delta_{2}, \delta_{3} \in \mathbb{C}$, not all zero. Of course, at least one of $\delta_{2}, \delta_{3}$ is not 0 . With no loss of generality we may assume that $\delta_{2}=1$. If $\delta_{3}=0$, then $P A=-\delta_{1} P$, whence $A=(I-P) A+P A=(I-P) A-\delta_{1} I+\delta_{1}(I-P)$, which in turn implies that $A+\delta_{1} I=(I-P)\left(A+\delta_{1} I\right)$ is a finite rank operator. Thus, Lemma 4.15 yields the desired conclusion. Assume, therefore, that $\delta_{3} \neq 0$. Then we have

$$
\begin{aligned}
\delta_{3} B & =\delta_{3} P B+\delta_{3}(I-P) B=-\delta_{1} P-P A+\delta_{3}(I-P) B \\
& =-\delta_{1} I-A+(I-P)\left(A+\delta_{3} B+\delta_{1} I\right) .
\end{aligned}
$$

Therefore, $B$ is a sum of a scalar operator, a scalar multiple of $A$, and a finite rank operator. Hence it follows that $[[T, A+F], A]$ is quasi-nilpotent for any $T \in \mathcal{A}$, where $F$ is a finite rank operator commuting with $A$. We claim that $I, A$ and $A^{2}$ are locally linearly dependent modulo $\operatorname{Im} F$. If this were not true, there would exist a nonzero $x \in X$ and $T \in \mathcal{A}$ such that $T \mid \operatorname{Im} F=0, T x=0, T A x=0$ and $T A^{2} x=x$. But then $[[T, A+F], A] x=x$, a contradiction. By Theorem 2.2 it follows that there is a nonzero polynomial $p$ such that $p(A)$ has finite rank. This implies that $A$ is algebraic, which in turn yields that $B$ is algebraic [5, Lemma 4.2], so that Lemma 4.14 gives the desired result.

The proof of Theorem 4.1 is thereby complete.

We conclude the paper with an observation concerning the noncommutative case. Suppose that $A$ and $B$ satisfy $B A=B^{2}=0$ and $A B=\alpha B$ for some nonzero scalar $\alpha$. Then $[[T, B], A]^{3}=0$. This provides an example of a noncommuting pair of derivations $d$ and $g$ such that the range of the product $d g$ consists of nilpotents. It can be proved that on the algebra of $n$ by $n$ matrices for some small values of $n$ (we did it for $n=2,3,4$ ) this example is essentially the only possible "noncommutative" example. We leave as an open question whether this remains true in more general algebras (possibly in dense algebras of linear operators).

\section{REFERENCES}

[1] S.A. Amitsur, Generalized polynomial identities and pivotal monomials, Trans. Amer. Math. Soc. 114 (1965), 210-226. MR 30:3117

[2] B. Aupetit, A primer on spectral theory, Springer-Verlag, 1991. MR 92c:46001

[3] J. Bergen, Derivations in prime rings, Canad. Math. Bull. 26 (1983), 267-270. MR 85a:16019

[4] M. Brešar, Derivations mapping into the socle, II, Proc. Amer. Math. Soc. 126 (1998), 181-187. MR 98c:46088

[5] M. Brešar, P. Šemrl, Derivations mapping into the socle, Math. Proc. Camb. Phil. Soc. 120 (1996), 339-346. MR 96m:46085

[6] P. Fillmore, C. Laurie, and H. Radjavi, On matrix spaces with zero determinant, Linear and Multilinear Algebra 18 (1985), 255-266. MR 87c: 15032

[7] N. Jacobson, Structure of rings, Amer. Math. Soc. Colloq. Publ., Vol. 37, Amer. Math. Soc., Providence, R. I., 1956. MR 18:373d

[8] I. Kaplansky, Infinite Abelian groups, Ann Arbor, 1954. MR 16:444g 
[9] V. Müller, Kaplansky's theorem and Banach PI-algebras, Pacific J. Math. 141 (1990), 355361. MR 91k:46048

[10] E. Posner, Derivations in prime rings, Proc. Amer. Math. Soc. 8 (1957), 1093-1100. MR 20:2361

[11] V. Ptak, Commutators in Banach algebras, Proc. Edinburgh Math. Soc. 22 (1979), 207-211. MR 81e: 46055

[12] H. Radjavi and P. Rosenthal, Invariant Subspaces, Springer-Verlag, 1973. MR 51:3924

[13] A. M. Sinclair, Continuous derivations on Banach algebras, Proc. Amer. Math. Soc. 20 (1969), 166-170. MR 38:1530

[14] Yu. V. Turovskii, V. S. Shul'man, Conditions for the massiveness of the range of a derivation of a Banach algebra and of associated differential operators, Mat. Zametki 42 (1987), 305314; English transl., Math. Notes 42 (1987), 669-674. MR 89e:14051

Department of Mathematics, University of Maribor, PF, KorošKa 160, 2000 Maribor, SLOVENIA

E-mail address: bresar@uni-mb.sl

Department of Mathematics, University of Maribor, SF, Smetanova 17, 2000 Maribor, SLOVENIA

E-mail address: peter.semrl@uni-mb.sl 